\title{
Price Regulation in Oligopolistic Markets
}

\author{
Luis C. Corchón' ${ }^{1}$ and Félix Marcos $^{2}$ \\ ${ }^{1}$ Departamento de Economía, Universidad Carlos III, 28903 Madrid, Spain \\ ${ }^{2}$ Departamento de Fundamentos del Análisis Económico, Universidad Complutense de Madrid, 28223 Madrid, Spain
}

Correspondence should be addressed to Luis C. Corchón, lcorchon@eco.uc3m.es

Received 15 September 2012; Accepted 8 November 2012

Academic Editors: C. Le Van and E. Yeldan

Copyright () 2012 L. C. Corchón and F. Marcos. This is an open access article distributed under the Creative Commons Attribution License, which permits unrestricted use, distribution, and reproduction in any medium, provided the original work is properly cited.

We consider price regulation in oligopolistic markets when firms are quantity setters. We consider a market for a homogeneous good with a demand function of special form ( $\rho$-linearity), constant returns to scale, and identical firms. Marginal costs can take two values only: low or high. Values of all parameters except the marginal costs are known to the regulator. Assuming that the regulator is risk-neutral and maximizes expected social welfare (defined as the sum of consumer surplus and profits), we characterize the optimal policy and show how this policy depends on the basic parameters of demand and costs.

\section{Introduction}

One of the main causes of market failure is lack of competition. Thus, a completely informed and benevolent regulator should be able to find an allocation in which all agents are better off than under oligopolistic competition. A great deal of attention has been devoted to the case in which the regulator lacks sufficient information to implement efficient allocations and a single firm supplies the whole market (see the survey by [1] and the references therein). In contrast, the case of regulation under an oligopoly has been relatively neglected. All the papers we are aware of are generalizations of optimal regulatory schemes that were proposed for the case of a monopoly: Shaffer [2] and Kim and Chang [3] generalized the work of Loeb and Magat [4], Schwermer [5] and Lee [6] generalized the work of Sappington and Sibley [7], and López-Cuñat [8] generalized the work of Baron and Myerson [9].

In this paper, we investigate the performance of a particular mechanism, price regulation, under conditions of oligopolistic competition and compare the welfare properties under its implementation with those of oligopolistic competition, also referred to as the free market (Our model does not answer the question of the optimal mechanism. In fact, Bertrand competition among firms will attain first best results. So in our model, Bertrand competition cannot be enforced by the regulator. For instance, the regulator might enforce price competition among firms but if firms condition prices on outputs, the Cournot competition will result [10]. Another interpretation of our work is that we study the properties of a particular mechanism that has been used in reality). The motivation for our study is that price regulation is (or has been) used to regulate oligopolistic markets such as gasoline, natural gas, electric power generation, telecommunications, healthcare, pharmaceuticals, and so forth. Again, the initial work was done for the case of a regulated monopoly; see Littlechild [11]. Price regulation is presented in textbooks as an inefficient mechanism under conditions of perfect competition. In this paper, we attempt to bridge monopoly and perfect competition by considering the intermediate case, namely, oligopoly (but note that all our results are still valid in the monopoly case). We will see that, under oligopoly, price regulation is, in some cases, more efficient than the free market.

In order to keep the analysis as simple as possible, we assume a very stylized scenario, as presented in Section 2. The product is homogeneous. The representative consumer is characterized by a $\rho$-linear demand curve (see [12]), which is known by the regulator. This functional form is parameterized by the real number $\rho$, which lies between -1 and infinity. When $\rho$ equals 1 , we have a linear demand, and, when $\rho$ is negative, we have a generalization of 
isoelastic demand. The industry is composed of a given number of identical firms with constant marginal costs; that is, we assume that costs are perfectly correlated (In this case, full extraction of the surplus can be achieved by the optimal mechanism; see Crémer and McLean $[13,14]$ for a generalization of earlier work by Maskin and Riley [15] and Myerson [16]. See Armstrong et al. [17] for an application to regulation.) The assumption that firms are identical is useful for distinguishing the problem of regulating a market where several firms have inefficient technologies (and so, in an optimal allocation, these firms must be eliminated) from the problem of pure regulation of an oligopoly. The marginal cost is allowed to take two values, high $\left(c_{H}\right)$ and low $\left(c_{L}\right)$. Firms know the true value of the marginal cost and, if not regulated, compete à la Cournot. In contrast, the regulator only knows the probabilities of occurrence of the two values of the marginal cost. The regulator chooses either a regulated price or allows for a free market (i.e., Cournot equilibrium). The regulator does not make transfers as in the standard regulation models, so the cost of public funds (see [18]) is not an issue here. The regulator is risk-neutral and maximizes expected social welfare, defined as the sum of consumer surplus and profits. Thus, in our model, consumers and firms are weighted identically.

In Section 3, we investigate regulation. We show that the optimal policy is either a free market or a regulated price equal to one of the two values of the marginal cost (Lemma 1). This is an interesting result because it simplifies the task of finding the optimal policy. According to this result, the optimal regulation is a tradeoff between the risky option, represented by $c_{L}$, which could be very profitable for the planner but risks zero output should real marginal cost be $c_{H}$, and the safe option, represented by $c_{H}$, which is always associated with positive production but is not optimal should the real marginal cost be $c_{L}$. (A similar tradeoff occurs in Vogelsang [19], but it applies to investment. The fact that high-cost firms might be shutdown under price regulation is also a feature of the optimal mechanism. For the monopoly case, see Baron and Myerson [9], p. 928, and Laffont and Martimort [20], Section 2.6.3).

In Sections 4 and 5, we show how the optimal policy depends on the underlying parameters. Instead of comparing the expected social welfare under a free market with that under regulation, we compare percentages of welfare losses. This is because the percentage of welfare loss under a free market does not depend on either the marginal costs or the intercept of the demand function, so the analysis is simplified greatly (Lemma 2).

We show that the optimal policy depends on four variables:

(i) the number of firms $(n)$,

(ii) the ratio of probabilities of occurrence of high and low marginal costs $(q)$,

(iii) a measure of the ratio between high and low marginal costs $(\beta)$,

(iv) the parameter $\rho$ of the demand function.
The role of the first three variables is what intuition suggests. Thus, the desirability of regulation decreases with the number of competitors (see Remark 5). In addition, for sufficiently small or large values of $q$, regulation is the optimal policy-with the regulated price equal to $c_{H}$ (resp., $c_{L}$ ) when this marginal cost is very likely because, in this case, the regulator faces very little uncertainty. For intermediate values of $q$, the free market may be socially optimal. Moreover, the values of $q$ for which any of these options is optimal form an interval (Proposition 6). With respect to $\beta$, when the high and low marginal costs are similar, the optimal policy is to set a regulated price equal to the higher value of the marginal cost. This is because this choice yields almost as much social welfare as the lower marginal cost and avoids the risk that firms do not produce. On the contrary, when the two marginal costs are sufficiently far apart, the optimal policy is to set a regulated price equal to the lower value of the marginal cost because this option yields greater social welfare. For intermediate values of $\beta$, the free market may or may not be the optimal policy. Again, the values of $\beta$ for which any of these options are optimal form an interval (Propositions 7 and 8) (however, $\beta$ also depends on the intercept of the demand function; see our comments below).

Finally, we look at the influence of $\rho$ on the optimal policy. Unfortunately, in this case we have been not able to fully define the optimal policy. We have only been able to characterize the intervals in which, should regulation occur, the price should be set either to $c_{H}$ or $c_{L}$. In particular, for values of $\rho$ close to 0 , the optimal policy is regulation with $p=c_{L}$. When $\rho$ tends to infinity, the optimal policy is regulation with $p=c_{H}$ (Propositions 9 and 10). In the first case, the optimal policy, should regulation occur, is to set the regulated price equal to the lower level of the marginal cost because demand is very elastic. In the second case, should regulation occur, the optimal policy is to set the regulated price equal to the higher value of the marginal cost because demand is very rigid.

Our results can be used to judge the success (or lack thereof) of price regulation in practice. In particular, they stress the importance of obtaining a good estimate of the intercept of the demand function and $\rho$. Since these values cannot be discerned by looking at points around equilibrium, price regulation in practice might be more difficult than presented in this paper.

Finally, Section 6 presents our final comments on the limitations of our work and mentions some possible extensions to consider price caps, several constant marginal costs, nonconstant marginal costs, and other forms of demand. We show that our previous results can be extended somewhat to price caps and several constant marginal costs but not to nonconstant marginal costs and other forms of demand.

\section{The Model}

There is a representative consumer with a utility function, $U=A x-b x^{\rho+1} /(\rho+1)+M$, where $x$ is aggregate output, $M$ is the consumption of the outside good, and $p$ is the market 
price. We assume $b \rho>0$ and $\rho>-1$ ( $\rho$ must be larger than -1 because if $\rho<-1$, utility tends to negative infinity if $x$ tends to 0 ). Given the budget constraint, $p x+M=I$ (where $I$ is the exogenous income), utility can be written as $U=A x-b x^{\rho+1} /(\rho+1)-p x$. Maximization of utility yields an inverse demand function $p=A-b x^{\rho}$. Because $b \rho>0$, $p$ is decreasing on $x$. Note that if $\rho<0, b<0$, and $A=0$, we have an isoelastic demand function $p=-b x^{\rho}$, and if $\rho=1$, demand is linear. Note also that there is a relationship between the demand elasticity (denoted by $\eta$ ) and $\rho$; namely, $\eta=-(d x / d p)(p / x)=p /(\rho(A-p))$. When $\rho<0, b<0$, then $A<p$ and $\eta$ increases with $\rho$. When $\rho>0, b>0$, then $A>p$ and $\eta$ decreases with $\rho$.

There are $n$ identical firms producing a single output denoted by $x_{i}$. Thus, $x=\sum_{i=1}^{n} x_{i}$. Marginal costs are constant and denoted by $c$. Defining $a=A-c$, profits for firm $i$ can be written as $B_{i}=\left(a-b x^{\rho}\right) x_{i}$. Assume $a b>0$, which implies that positive outputs are possible. If firms are quantity setters, it is easy to check that second-order conditions of profit maximization hold and that the equilibrium is symmetric. Thus, first-order conditions of profit maximization yield the Cournot equilibrium outputs and profits, denoted by the superscript $c$; namely,

$$
\begin{gathered}
x^{C}=\left[\frac{a n}{b(n+\rho)}\right]^{1 / \rho}, \\
B_{i}^{C}=b \rho n^{(1-\rho) / \rho}\left[\frac{a}{b(n+\rho)}\right]^{(1+\rho) / \rho} .
\end{gathered}
$$

Social welfare, denoted by $W$, is the sum of profits and the utility of the representative consumer; that is,

$$
W=U+\sum B_{i}=a x-\frac{b x^{\rho+1}}{\rho+1} .
$$

Social welfare in the Cournot equilibrium, denoted by $W^{C}$, is

$$
W^{C}=\frac{a^{(1+\rho) / \rho} \rho n^{1 / \rho}(n+\rho+1)}{b^{1 / \rho}(n+\rho)^{(1+\rho) / \rho}(\rho+1)} .
$$

In an optimal allocation, social welfare is maximized. Since the second-order condition holds and the allocation is symmetric, the first-order condition of social welfare maximization yields the optimal allocation; namely,

$$
x^{O}=\left(\frac{a}{b}\right)^{1 / \rho}, \quad W^{O}=\frac{\rho a^{(1+\rho) / \rho}}{b^{1 / \rho}(1+\rho)} .
$$

\section{Regulation}

We assume that the regulator controls the market price. The regulator is uncertain of the value of marginal cost, which can take two values: $c_{H}$ with probability $\pi$ and $c_{L}$ with probability $1-\pi$. We will refer to these values as "states of the world." Once the regulated price is set, firms decide on output. If $p$ is smaller than the value of the marginal cost, firms do not produce and social welfare is zero. If $p$ is larger than or equal to the marginal cost, they produce an identical quantity each $x_{i}=x / n$, satisfying demand; that is, $x=((A-p) / b)^{1 / \rho}$. Thus, social welfare in state $c=c_{H}, c_{L}$ with regulated price $p$ is

$$
\begin{array}{rl}
W^{R}=A & x-\frac{b x^{\rho+1}}{\rho+1}-c x=\left(\frac{A-p}{b}\right)^{1 / \rho} \\
& \times\left(\frac{\rho A+p-(1+\rho) c}{1+\rho}\right) \quad \text { if } x>0 . W^{R}=0, \text { otherwise. }
\end{array}
$$

We assume that the regulator is risk-neutral and maximizes expected social welfare. Firstly, we show that, in this case, the regulator chooses either $p=c_{H}$ or $p=c_{L}$.

Lemma 1. A regulator maximizing expected social welfare chooses a price of $c_{H}$ or $c_{L}$.

Proof. Let $U(x)$ be the utility function of the representative consumer and $x(p)$ the demand at price $p$. Thus, if $x>0$, social welfare can be written as $W=U(x(p))-c x(p)$. Differentiating this equation, we see that, if $p>c$, social welfare decreases in $p$.

Suppose that $p>c_{H}$. Expected social welfare can be increased by decreasing $p$, so this price cannot be optimal. Suppose now that $c_{L}<p<c_{H}$. If $c=c_{H}$, a decrease in the regulated price has no effect on social welfare, but it increases social welfare if $c=c_{L}$. Therefore, a price decrease improves expected social welfare. Since the regulator will never choose a price below $c_{L}$, the lemma is proved.

The reasoning behind Lemma 1 is simple. The regulator wants prices to be as low as possible. However, if too low a price is selected, the risk is that the industry will not produce at all. Thus, if the regulator chooses a price that is larger than $c_{H}$ (resp., $c_{L}$ ), there is as much risk of zero output as choosing $p=c_{H}$ (resp., $c_{L}$ ) but, if costs are $c_{H}$ (resp., $c_{L}$ ), the result is not as good as when the price is set equal to $c_{H}$ (resp., $c_{L}$ ).

Lemma 1 implies that the regulated price is either $c_{H}$ or $c_{L}$. The expected welfare in these two cases is easily shown as follows:

$$
\begin{aligned}
E W^{H}= & \frac{\rho \pi}{(1+\rho) b^{1 / \rho}}\left(A-c_{H}\right)^{(1+\rho) / \rho} \\
& +(1-\pi)\left(\frac{A-c_{H}}{b}\right)^{1 / \rho} \\
& \times \frac{\rho\left(A-c_{L}\right)-\left(A-c_{H}\right)+\left(A-c_{L}\right)}{1+\rho}, \\
E W^{L} & =(1-\pi)\left(\frac{A-c_{L}}{b}\right)^{1 / \rho} \frac{\rho\left(A-c_{L}\right)}{1+\rho} \\
& =\frac{\rho(1-\pi)}{b^{1 / \rho}(1+\rho)}\left(A-c_{L}\right)^{(1+\rho) / \rho} .
\end{aligned}
$$

The regulator chooses $p=c_{H}$ if $E W^{H}>E W^{L}$, and $p=c_{L}$ otherwise. The regulator is indifferent to the price if $E W^{H}=E W^{L}$. Define $a_{H}=A-c_{L}, a_{L}=A-c_{H}, \beta=a_{H} / a_{L}$, 
and $q=\pi / 1-\pi$. Note that if $\rho>0, \beta>1$ and if $\rho<0$, $0<\beta<1$, (the case of $\beta=1$, namely, $c_{H}=c_{L}$, is trivial and is not considered). With this notation in hand, $E W^{H}=E W^{L}$ is equivalent to

$$
q=\frac{\rho \beta\left(\beta^{1 / \rho}-1\right)-\beta+1}{\rho}
$$

\section{Comparing the Free Market with Regulation}

In order to compare the welfare expected under free market conditions (e.g., Cournot competition) with the social optimum, we will find it more convenient to work in terms of percentages of welfare losses. As we will see, this greatly simplifies our analysis. Define the percentage of welfare loss, denoted by PWL, of an allocation yielding, say, social welfare $W^{\prime}$, as follows:

$$
\mathrm{PWL}=\frac{W^{O}-W^{\prime}}{W^{O}} .
$$

The percentage of welfare loss under the Cournot competition, denoted by $\mathrm{PWL}^{C}$, is

$$
\mathrm{PWL}^{C}=1-\frac{n^{1 / \rho}(n+\rho+1)}{(n+\rho)^{(1+\rho) / \rho}}=\operatorname{PWL}^{C}(\rho, n) .
$$

We now review the (well-known) properties of $\mathrm{PWL}^{C}$. Clearly, $W^{C}$ increases with $n$. Since $W^{O}$ is independent of $n$, PWL ${ }^{C}$ decreases with $n$. In addition, an easy adaptation of Proposition 6 in Andersen and Renault [12] shows that $\mathrm{PWL}^{C}$ is strictly quasiconcave on $\rho$, with a maximum value that decreases with $n$ and that, for the duopoly case, is 0.118 . Finally, $\mathrm{PWL}^{C}$ tends to 0 when $\rho$ tends to -1 or to $\infty$.

First, from (5) and (6) we obtain the percentage of the expected welfare losses from price regulation. The optimal expected welfare, denoted by $E W^{0}$, is the expected social welfare obtained if, for each state of the world, the planner had a complete set of information; that is, when $c=c_{H}$ (resp., $\left.c=c_{L}\right)$, the regulated price would be $c_{H}$ (resp., $\left.c_{L}\right)$. Thus,

$$
E W^{0}=\frac{\rho}{b^{1 / \rho}(1+\rho)}\left(\pi a_{L}^{(1+\rho) / \rho}+(1-\pi) a_{H}^{(1+\rho) / \rho}\right) .
$$

Next, we note that the percentage of expected welfare losses from a Cournot equilibrium is equal to the percentage of welfare losses obtained in (9) above. The reasoning behind this result is that the parameter, $a$, which encapsulates all the uncertainties faced by the regulator, multiplicatively affects social welfare both at the Cournot and the optimal allocation (see (3) and $\left.\left(2^{\prime}\right)\right)$. Consequently, a cancels out in the formula governing the percentage of expected welfare loss. We record this result as Lemma 2 below.

Lemma 2. The percentage of expected welfare loss under the Cournot competition equals the percentage of welfare loss.

The proof involves only cumbersome algebra and is left to the reader. Subsequently, we will denote the percentage of expected welfare loss under the Cournot competition by
$\mathrm{PWL}^{C}$. We are now ready to compare free market and price regulation scenarios.

(1) Consider first that the regulator chooses $p=c_{H}$. We will compare the percentage of welfare losses from price regulation when $p=c_{H}$, denoted by $\mathrm{PWL}^{H}$, with the welfare losses under the Cournot competition:

$$
\begin{aligned}
\mathrm{PWL}^{H} & =\frac{E W^{0}-E W^{H}}{E W^{0}}=\frac{\rho \beta^{(1+\rho) / \rho}-\rho \beta-\beta+1}{\rho\left(q+\beta^{(1+\rho) / \rho}\right)} \\
& =\operatorname{PWL}^{H}(\rho, \beta, q) .
\end{aligned}
$$

Next, Lemma 3 (proved in the Appendix) shows the properties of $\mathrm{PWL}^{H}$.

Lemma 3. $\mathrm{PWL}^{H}(\bullet)$ is decreasing in $\rho$ and increasing in $\beta$ if $\rho>0$ (increasing in $\rho$ and decreasing in $\beta$ if $\rho<0$ ) and decreasing in $q$.

Comparing (11) with the welfare losses under the Cournot equilibrium, easy calculations show that $\mathrm{PWL}^{C}=$ $\mathrm{PWL}^{H}$ iff

$$
q=\frac{(\rho \beta+\beta-1)(n+\rho)^{(1+\rho) / \rho}-\rho \beta^{(1+\rho) / \rho}(n+\rho+1) n^{1 / \rho}}{\rho n^{1 / \rho}(n+\rho+1)-\rho(n+\rho)^{(1+\rho) / \rho}} .
$$

(2) Now consider that the regulator chooses $p=c_{L}$. The expected welfare is

$$
E W^{L}=(1-\pi)\left(\frac{A-c_{L}}{b}\right)^{1 / \rho} \frac{\rho\left(A-c_{L}\right)}{1+\rho}=\frac{(1-\pi) \rho}{b^{1 / \rho}(1+\rho)} a_{H}^{(1+\rho) / \rho} .
$$

The percentage of welfare losses from price regulation when $p=c_{L}$, denoted by $\mathrm{PWL}^{L}$, is

$$
\begin{aligned}
\mathrm{PWL}^{L} & =1-\frac{(1-\pi) a_{H}^{(1+\rho) / \rho}}{\pi a_{L}^{(1+\rho) / \rho}+(1-\pi) a_{H}^{(1+\rho) / \rho}} \\
& =\frac{q}{q+\beta^{(1+\rho) / \rho}}=\operatorname{PWL}^{L}(\rho, \beta, q) .
\end{aligned}
$$

We now investigate the properties of $\mathrm{PWL}^{L}$. The proof of the lemma is trivial and is omitted.

Lemma 4. $\operatorname{PWL}^{L}(\bullet)$ is decreasing in $\beta$ if $\rho>0$ (increasing if $\rho<0$ ), increasing in $\rho$ if $\rho>0$ (decreasing if $\rho<0$ ) and increasing in $q$.

Similar to the aforementioned Case (1), the value of $q$ equalizing welfare losses under regulation with $p=c_{L}$ and the Cournot equilibrium is

$$
q=\frac{\beta^{(1+\rho) / \rho}\left[(n+\rho)^{(1+\rho) / \rho}-n^{1 / \rho}(n+\rho+1)\right]}{n^{1 / \rho}(n+\rho+1)} .
$$

Note that, when $\beta$ tends to $1, \mathrm{PWL}^{H}$ tends to 0 but $\mathrm{PWL}^{L}$ tends to $q /(q+1)$. The reason for that is the existence of a discontinuity: if the state of the world is $c_{H}$ but the regulator 


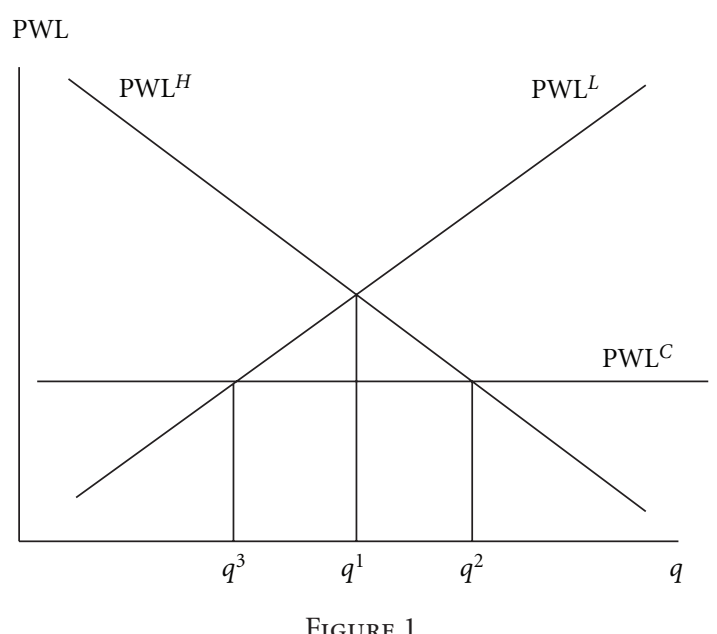

Figure 1

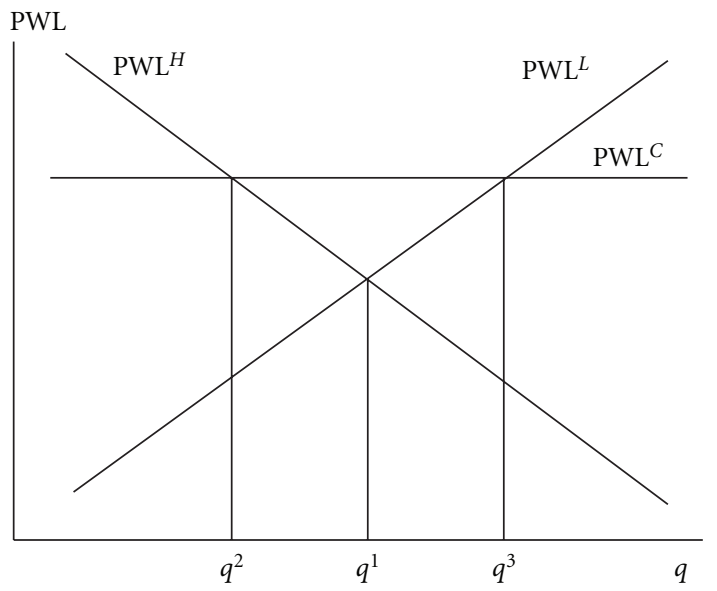

Figure 2 set $p=c_{L}$, there is no production so the welfare loss is positive even for $c_{H} \simeq c_{L}$. However, if the regulator set $p=c_{H}$, the welfare loss tends to 0 when $c_{H}$ tends to $c_{L}$.

Summing up, in order to find the optimal policy, a regulator has to look at three equations only, namely, (9), (11), and (14).

\section{The Optimal Policy: Comparative Statics}

In this section, we characterize the values of $\rho, \beta, n$, and $q$ for which a regulation or a free market is the optimal choices. We have two kinds of results: results that do not depend on the sign of $\rho$ and results that depend on its sign (recall that, when $\rho>0$, the demand function is concave or convex depending on whether $\rho$ is larger or smaller than 1 , respectively. When $\rho<0$, the demand function is convex). In the first class, we consider the characterization of the optimal policy when $n$ and $q$ vary. In the second class, we consider the characterization of the optimal policy when $\rho$ and $\beta$ vary. Firstly, we note the following remark.

Remark 5. Given $\rho, \beta$, and $q$ there is an $n^{1}$ such that if $n \in$ $\left[1, n^{1}\right]$, the optimal policy is regulation (either with $p=c_{L}$ or with $p=c_{H}$ ). If $n>n^{1}$, the optimal policy is free market.

The remark follows from the fact that, as we remarked after (5), the welfare loss under the Cournot competition decreases with $n$ and tends to zero when $\mathrm{n}$ tends to infinity and that the expected welfare loss under regulation does not depend on $n$. This remark is just a restatement of the wellknown fact that, given identical firms with no economies of scale, the more the competition in the market, the better. Clearly, when $n^{1}$ is 1 , the interval $\left(1, n^{1}\right]$ is empty and a free market is always the optimal policy.

Now we turn to the study of the optimal policy when $q$ varies. For given values of $n, \rho$, and $\beta$, denote by $q^{1}$ the value of $q$ in (7). Similarly, define $q^{2}$ and $q^{3}$ as the values of $q$ given by (12) and (15), respectively. See Figures 1 and 2.

Proposition 6. Let $\rho, \beta$, and $n$ be given. Suppose that $q^{2} \geq q^{3}$. If $q \in\left(0, q^{3}\right]$, the optimal policy is regulation with $p=c_{L}$. If $q \in\left[q^{3}, q^{2}\right]$, the optimal policy is a free market. If $q \in\left[q^{2}, \infty\right)$, the optimal policy is a regulation with $p=c_{H}$. Suppose that $q^{2}<q^{3}$. If $q \in\left(0, q^{1}\right]$, the optimal policy is a a regulation with $p=c_{L}$. If $q \in\left[q^{1}, \infty\right)$, the optimal policy is a regulation with $p=c_{H}$.

Proof. Note the following facts.

(a) $\operatorname{PWL}^{H}(\rho, \beta, \bullet)$ is decreasing in $q$ as shown by Lemma 3 and tends to 0 when $q$ tends to infinity (see (11)).

(b) $\operatorname{PWL}^{L}(\rho, \beta, \bullet)$ is increasing in $q$ as shown in Lemma 4 , tends to 0 when $q$ tends to 0 , and tends to 1 when $q$ tends to infinity (see (14)).

(c) $\mathrm{PWL}^{C}$ is larger than 0 and does not depend on $q$ (see (9)).

Note that (a) and (b) above imply that $q^{1}$ and $q^{3}$ exist. Then, the proposition follows from (a), (b), and (c) by noting that the values of $q$ are defined by the equality between PWL at regulated prices $c_{H}$ or $c_{L}$ and the Cournot equilibrium.

The reasoning behind Proposition 6 is as follows. When $q$ is small enough, the optimal policy is a regulation with $p=c_{L}$ because the probability of $c=c_{L}$ is very large so the welfare loss of regulation is negligible because the case $c=c_{H}$ seldom occurs ( $\mathrm{PWL}^{L}$ is close to 0 ). When $q$ increases, on the one hand, $\mathrm{PWL}^{L}$ increases because the probability of $c=c_{H}$ increases (in this case there is no production), and, on the other hand, $\mathrm{PWL}^{H}$ decreases for the same reason. Thus, for the intermediate value theorem, there is a value of $q\left(q^{1}\right)$ for which $\mathrm{PWL}^{L}=\mathrm{PWL}^{H}$. If, for $q^{1}, \mathrm{PWL}^{H}<\mathrm{PWL}^{C}$, the regulation is always better because $\mathrm{PWL}^{H}$ is decreasing in $q$ and tends to 0 when $q$ tends to infinity. On the contrary, if, for $q^{1}, \mathrm{PWL}^{H}>\mathrm{PWL}^{C}$, there is an interval $\left[q^{2}, q^{3}\right]$ where a free market is the optimal policy.

We now turn our attention to the optimal policy when $\beta$ or $\rho$ varies. We have to consider two cases: when $\rho$ is positive and $\beta$ lies between 1 and infinity and when $\rho$ is negative and $\beta$ lies between 0 and 1 . 
PWL

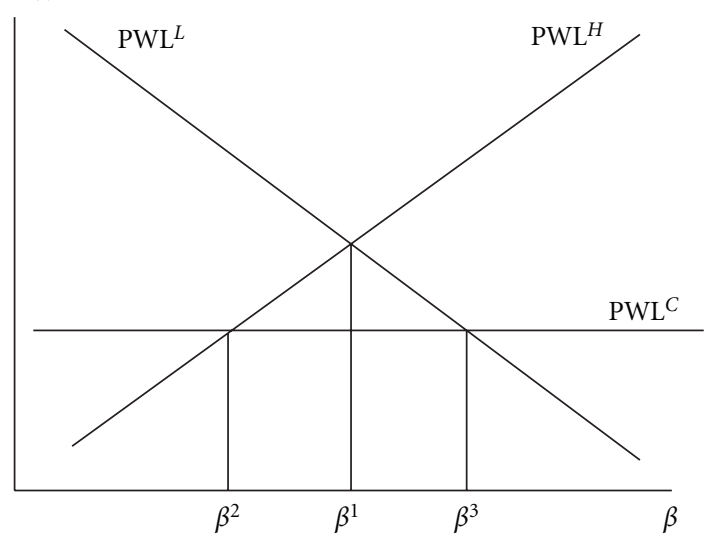

Figure 3

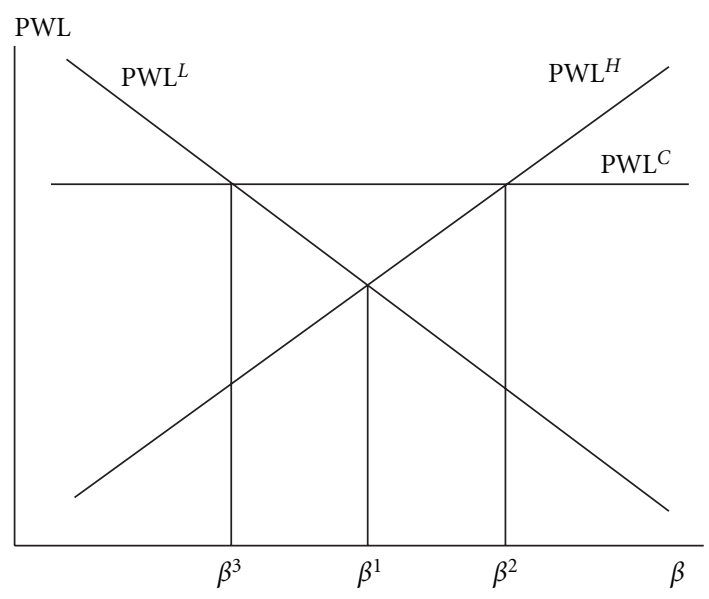

FIGURE 4

Let $\rho$ and $q$ be given. Let $\beta^{1}$ be the value of $\beta$ solving $\operatorname{PWL}^{H}(\rho, \beta, q)=\operatorname{PWL}^{L}(\rho, \beta, q)$; that is, it solves (7). Let $\beta^{2}$ be the value of $\beta$ solving $\operatorname{PWL}^{C}(\rho, n)=\operatorname{PWL}^{H}(\rho, \beta, q)$; that is, it solves (12). $\beta^{1}$ and $\beta^{2}$ exist and are unique (see the proof of the next proposition). Let $\beta^{3}$ be the value of $\beta$ solving $\operatorname{PWL}^{C}(\rho, n)=\operatorname{PWL}^{L}(\rho, \beta, q)$; that is, it solves (15). If such a value exists, it is unique. If this value does not exist, set $\beta^{3}=1$ (the lower bound for $\beta$ ). See Figures 3 and 4 below.

Proposition 7. Suppose that $\rho>0$ and $n$ and $q$ are given. Suppose that $\beta^{3}>\beta^{2}$. If $\beta \in\left(1, \beta^{2}\right]$, the optimal policy is a regulation with $p=c_{H}$. If $\beta \in\left[\beta^{2}, \beta^{3}\right]$, the optimal policy is a free market. If $\beta \in\left[\beta^{3}, \infty\right)$, the optimal policy is a regulation with $p=c_{L}$. Suppose that $\beta^{2}>\beta^{3}$. If $\beta \in\left(1, \beta^{1}\right]$, the optimal policy is a regulation with $p=c_{H}$. If $\beta \in\left[\beta^{1}, \infty\right)$, the optimal policy is a regulation with $p=c_{L}$.

Proof. Note the following facts.

(a) $\mathrm{PWL}^{H}$ tends to 0 if $\beta$ tends to 1 and tends to 1 if $\beta$ tends to infinity (see $(11)$ ). $\operatorname{PWL}^{H}(\rho, \bullet, q)$ is increasing on $\beta$ as shown in Lemma 3.

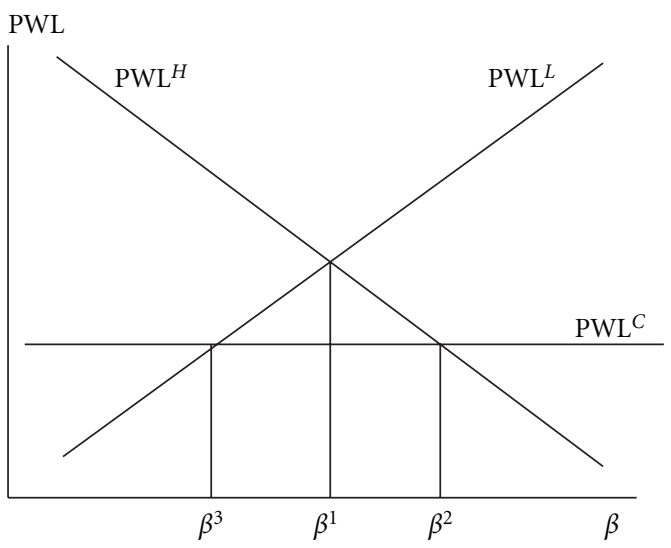

Figure 5

(b) $\mathrm{PWL}^{L}$ tends to $q /(q+1)$ if $\beta$ tends to 1 and tends to 0 if $\beta$ tends to infinity (see $(14))$. $\operatorname{PWL}^{L}(\rho, \bullet, q)$ is decreasing on $\beta$ as shown in Lemma 4.

(c) $\mathrm{PWL}^{C}$ does not depend on $\beta$ (see (9)).

Note that (a) and (b) above imply that $\beta^{1}$ and $\beta^{2}$ exist. The proposition then follows from (a), (b), and (c) by noting that the values of $\beta$ are defined by the equalities between PWL at regulated prices $c_{H}$ or $c_{L}$ and the Cournot equilibrium.

The reasoning behind Proposition 7 is as follows. When $\beta$ is close to 1 , a regulation is better than a free market because the regulator faces very little uncertainty since $c_{H}$ and $c_{L}$ are almost identical so that the expected welfare loss of regulation tends to 0 . In this case, the regulator sets $p=c_{H}$ because the welfare loss of setting $c_{H}$ when costs are $c_{L}$ is very small compared with the loss of setting $c_{L}$ when the cost is $c_{H}$ (in the latter case firms will not produce). When $\beta$ is large enough, regulation is better with $p=c_{L}$ because, when $c_{H}$ tends to $A$, the surplus of setting a regulated price of $c_{H}$ tends to 0 , so the welfare loss when $p=c_{L}$ and $c=c_{H}$ is very small. Thus, for the intermediate value theorem, there is a value of $\beta\left(\beta^{1}\right)$ for which $\mathrm{PWL}^{L}=\mathrm{PWL}^{H}$. If, for $\beta^{1}, \mathrm{PWL}^{L}<\mathrm{PWL}^{C}$, the regulation is always better because $\mathrm{PWL}^{L}$ is decreasing in $\beta$ and tends to 0 when $\beta$ tends to infinity. On the contrary, if, for $\beta^{1}, \mathrm{PWL}^{L}>\mathrm{PWL}^{C}$, there is an interval $\left[\beta^{2}, \beta^{3}\right]$ where a free market is the optimal policy.

The next proposition deals with the case in which $\rho \in$ $(-1,0)$. We keep the notation for $\beta$ introduced above and recall that here $\beta \in[0,1]$. See Figures 5 and 6 .

Proposition 8. Suppose that $\rho \in(-1,0), n$ and $q$ are given. Suppose that $\beta^{2}>\beta^{3}$. If $\beta \in\left(0, \beta^{3}\right]$, the optimal policy is a regulation with $p=c_{L}$. If $\beta \in\left[\beta^{3}, \beta^{2}\right]$, the optimal policy is a free market. If $\beta \in\left[\beta^{2}, 1\right)$, the optimal policy is a regulation with $p=c_{H}$. Suppose that $\beta^{3}>\beta^{2}$. If $\beta \in\left(0, \beta^{1}\right]$, the optimal policy is a regulation with $p=c_{L}$. If $\beta \in\left[\beta^{1}, 1\right)$, the optimal policy is a regulation with $p=c_{H}$.

Proof. Note the following facts.

(a) $\mathrm{PWL}^{H}$ tends to 0 if $\beta$ tends to 1 . $\operatorname{PWL}^{H}(\rho, \bullet, q)$ is decreasing on $\beta$ and tends to 1 if $\beta$ tends to 0 . 


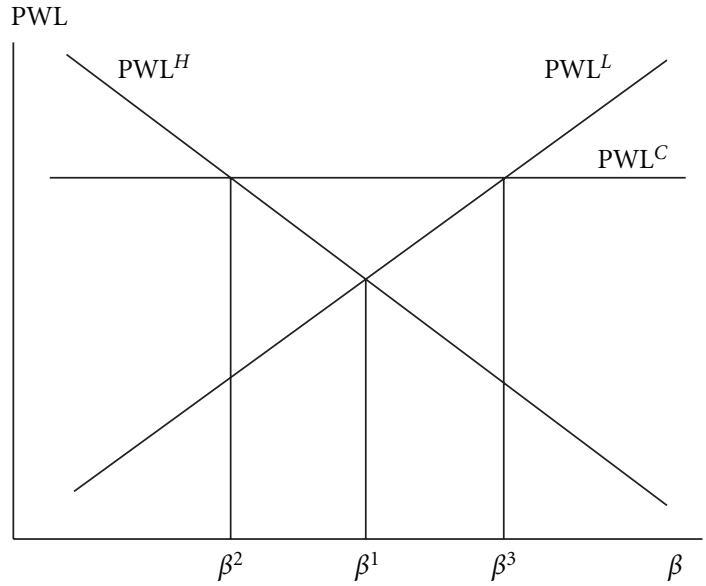

Figure 6

(b) $\mathrm{PWL}^{L}$ tends to $q /(q+1)$ if $\beta$ tends to $1 . \operatorname{PWL}^{L}(\rho, \bullet, q)$ is increasing on $\beta$ and tends to 0 if $\beta$ tends to 0 .

(c) $\mathrm{PWL}^{C}$ does not depend on $\beta$.

The proposition follows from (a), (b), and (c) by noting that the values of $\beta$ are defined by the equalities between PWL at regulated prices $c_{H}$ or $c_{L}$ and the Cournot equilibrium.

The reasoning behind Proposition 8 is as follows. When $\beta$ is small enough, regulation is better with $p=c_{L}$ because the surplus when $c=c_{L}$ is very large and the welfare loss when $c=c_{H}$ is relatively negligible. When $\beta$ is large enough, regulation with $p=c_{H}$ is the optimal policy because $c_{H}$ is close to $c_{L}$ and the welfare loss when $c=c_{L}$ is very small. On the contrary, if $p=c_{L}$, the welfare loss of $c=c_{H}$ can be very large because there is no production. When $\beta$ increases, $\mathrm{PWL}^{L}$ increases and $\mathrm{PWL}^{H}$ decreases towards 0 , so there is a value of $\beta\left(\beta^{1}\right)$ for which $\mathrm{PWL}^{L}=\mathrm{PWL}^{H}$. If, for $\beta^{1}, \mathrm{PWL}^{H}<$ $\mathrm{PWL}^{C}$, a regulation is always better. On the contrary, if, for $\beta^{1}, \mathrm{PWL}^{H}>\mathrm{PWL}^{C}$, there is room for a free market.

Propositions 7 and 8 say two things: firstly, when $c_{H}$ and $c_{L}$ are almost identical (so that $\beta$ is close to 1 ), a regulation with price equal to $c_{H}$ (i.e., the safe option because it secures a positive output, no matter what) is the optimal choice; secondly, when $c_{H}$ and $c_{L}$ are very different (so that $\beta$ is very large when $\rho$ is positive and close to 0 when $\rho$ is negative), a regulation with price equal to $c_{L}$ (i.e., the risky option) is the optimal choice given the greater social welfare that is created in this case. (The relationship between $\beta$ and the marginal costs is not straightforward because $\beta$ also depends on $A$. Thus the same values of the marginal costs might be associated with very different policies.)

Propositions 6, 7, and 8 imply that when $\beta$ or $q$ is small or large enough, regulation is always the optimal policy. This is very intuitive because in these cases, the uncertainty faced by the regulator disappears. Note that, when $\rho<0$, a price equal to $c_{H}$ is optimal for values of $\beta$ close to 1 (which is the largest value of $\beta$ ) and that, when $\rho>0$, this price is also optimal when $\beta$ is close to 1 (which is the smallest value of $\beta$ ).

Finally, we consider variations of $\rho$. This case is more complicated than the previous ones because $\mathrm{PWL}^{C}$ depends on $\rho$. Consequently, our conclusions are not as sharp as in the previous results. Let $\rho^{*}$ be such that $\operatorname{PWL}^{L}\left(\rho^{*}\right)=$ $\operatorname{PWL}^{H}\left(\rho^{*}\right)$.

Proposition 9. Suppose that $\beta, n$, and $q$ are given and that $\rho>0$. For $\rho$ close enough to 0 , the optimal policy is a regulation with $p=c_{L}$. When $\rho$ tends to infinity, the optimal policy is a regulation with $p=c_{H}$. Moreover, $c_{H}$ cannot be optimal for $\rho<\rho^{*}$ and $c_{L}$ cannot be optimal for $\rho>\rho^{*}$.

Proof. Note the followings facts.

(a) $\mathrm{PWL}^{C}$ is positive when $\rho \rightarrow 0$. $\mathrm{PWL}^{C}(\bullet, n)$ is quasiconcave with a maximum and decreasing when $\rho$ is large enough. When $\rho=1, \mathrm{PWL}^{C}>0$ and when $\rho \rightarrow$ $\infty, \mathrm{PWL}^{C} \rightarrow 0$ (see Andersen and Renault [12], p. 262).

(b) $\operatorname{PWL}^{L}(\bullet, \beta, q)$ is increasing with $\rho \cdot \mathrm{PWL}^{L} \rightarrow 0$ if $\rho \rightarrow$ 0 and $\mathrm{PWL}^{L} \rightarrow q /(q+\beta)$ when $\rho \rightarrow \infty$.

(c) $\operatorname{PWL}^{H}(\bullet, \beta, q)$ is decreasing with $\rho$. $\mathrm{PWL}^{H} \rightarrow 1$ if $\rho \rightarrow 0$ and $\mathrm{PWL}^{H} \rightarrow 0$ when $\rho \rightarrow \infty$.

(b) and (c) imply that $\rho^{*}$ exists and is unique.

From (a), (b), and (c) the proposition follows, noting that it can be shown that when $\rho \rightarrow \infty, \lim \mathrm{PWL}^{H} / \mathrm{PWL}^{C}=$ 0 .

The reasoning behind Proposition 9 is that, when $\rho$ is close to 0 , the demand function is very elastic, so the welfare gains in the state of the world $c=c_{L}$ are very large with respect to the welfare gains when the state of the world is $c=c_{H}$. For the same reason, a regulation with a price at $c_{L}$ is better than a free market. When $\rho$ is close to infinity, the demand function is almost rigid, so the total surplus is almost independent of the price and setting the regulated price at $c_{L}$ risks much of the welfare gain if the state of the world is $c_{H}$. In this case (even when the state of the world is $c_{L}$ ), the price under a free market tends to $A$ and the welfare losses are larger (maybe only by a tiny bit) with a free market than with a regulation with $p=c_{H}$.

Note that Proposition 9 does not characterize the optimal policy for intermediate values of $\rho$. However, it says that there is a cut-off value of $\rho, \rho^{*}$, such that under regulation, $c_{L}$ is optimal for all $\rho<\rho^{*}$ and $c_{H}$ is optimal for all $\rho>$ $\rho^{*}$. Unfortunately, we cannot prevent $\mathrm{PWL}^{C}$ intersecting the other two curves several times, so the optimal policy might switch from free market to regulation and back again to free market, and so forth, as $\rho$ increases. Our simulations for values of $q$ and $\beta$ that are not extreme could not prove this possibility, however.

Finally, we tackle the case in which $\rho$ is negative.

Proposition 10. Suppose that $\beta, n$, and $q$ are given and that $\rho<0$. For $\rho$ close enough to 0 , the optimal policy is a regulation with $p=c_{L}$. Moreover, $c_{L}$ cannot be optimal for $\rho<\rho^{*}$ and $c_{H}$ cannot be optimal for $\rho>\rho^{*}$.

Proof. This proof is virtually identical to Proposition 9.

The reasoning behind Proposition 10 is that $\operatorname{PWL}^{L}(\beta, \bullet, q) \rightarrow 0$ if $\rho \rightarrow 0$ but the limits of $\mathrm{PWL}^{H}$ 
and $\mathrm{PWL}^{C}$ are not 0 , so regulation with $p=c_{L}$ is the optimal policy. The reason is that the demand is very elastic. This is different from the result in Proposition 9 aforementioned because, there, $\beta$ was larger than 1 and, in this case, $\beta$ is between 0 and 1 . When $\rho \rightarrow-1 \operatorname{PWL}^{L}(\beta, \bullet, q) \rightarrow q / q+1$ but $\operatorname{PWL}^{H}(\beta, \bullet, q)$ and $\operatorname{PWL}^{C}(\beta, \bullet, q)$ tend to 0 , so regulation with $p=c_{L}$ cannot be the optimal policy. The reason is that, when $\rho \rightarrow-1$, the demand function tends to be of elasticity 1 and this function yields an infinite surplus, so the policy of setting a regulated price of $c_{L}$ is very risky. Depending on the parameters, the optimal policy could be a regulation with $p=c_{H}$ or a free market. For instance, when $\beta=0.4$, $q=0.1$, and $n=10$, a free market is optimal. The reason is that when the two values of the marginal costs are far apart, the probability of occurrence of $c_{H}$ is small and competition is high. When $\beta=0.9, q=10$, and $n=2$, the regulation is optimal. The reason is that marginal costs are close, the probability of occurrence of $c_{H}$, is large and competition is between the two firms only.

\section{Extensions and Final Comments}

In this paper, we have studied, in a very simple framework, the optimal regulatory policy in an oligopolistic market where marginal costs are unknown to the regulator. There are many aspects of paramount practical importance that have been left out of our study because they would require fresh modelling: that is, quality of the product, location, repeated interaction among firms and the regulator, different objectives for the regulator (i.e., proconsumer or profirms), a continuous distribution of marginal costs, or variability of demand (for the latter case, see Earle et al. [21]). Other aspects could be incorporated or, at least, discussed in our framework such as the four points considered below.

(1) Price Caps. In many practical applications, instead of setting a price, the regulator sets a price cap, that is, a ceiling for the price (an analysis of the investment incentives under price caps has been done by Fabra et al. [22] and by Buehler et al. [23]). In our case, this means that, once the regulated price has been set, say to $k$, the regulator would allow for any increase in output starting from $p^{-1}(k) / n$. We will see that, if $\rho>0, \beta-1 \leq \rho / n$ and if $\rho<0, \beta-1 \geq \rho / n$, price caps coincide with regulated prices. Indeed, suppose that the regulated price is $c_{L}$. If the true cost is $c_{H}$, production is zero and no firm has an incentive to increase production because costs are never recovered for any positive output. If the true cost is $c_{L}$ and a firm increases output, it will face losses. Now, suppose that the regulated price is $c_{H}$. Let $x\left(c_{H}\right)$ be the optimal output when the regulated price is $c_{H}$; that is, $x\left(c_{H}\right)=p^{-1}\left(c_{H}\right)$. If the true cost is $c_{H}$, again, if a firm increases output, it will face losses. So, we are left with the case in which the true cost is $c_{L}$. Any infinitesimal increase in the output of firm $i$ must not increase profits; namely,

$$
\frac{d \pi_{i}}{d x_{i}}=\frac{d p\left(x\left(c_{H}\right)\right)}{d x} \cdot \frac{x\left(c_{H}\right)}{n}+c_{H}-c_{L} \leq 0
$$

In fact, if (16) holds, because of the concavity of the profit function, we guarantee that firm $i$ would not choose to increase output, infinitesimally or not. Noting that $c_{H}-c_{L}=$ $a_{H}-a_{L}$ and calculating $d p\left(x\left(c_{H}\right)\right) / d x$ and $x\left(c_{H}\right)$ from $\left(2^{\prime}\right)$, we obtain from (16) that

$$
\left(a_{H}-a_{L}\right) n \leq \rho a_{L} .
$$

Recall that $\beta=a_{H} / a_{L}$. Now we have two cases. If $\rho$ is positive, $a_{L}$ is positive and inequality (17) is equivalent to $\beta-1 \leq \rho / n$. If $\rho$ is negative, $a_{L}$ is negative and inequality (17) is equivalent to $\beta-1 \geq \rho / n$. This says that, if costs do not display too much variability, firms will not take the opportunity to increase their output when the regulated price is $c_{H}$ and the state of the world is $c_{L}$. This is because the values of marginal costs are not sufficiently far apart to justify an increase in output. For instance, assume that there are four firms in the market. If the demand is linear, $\beta$ should be smaller than $(n+1) / n$. This implies that the variability in $a$ must be less than $25 \%$. When demand is isoelastic with, say, elasticity of $1.5, \rho$ equals 0.66 . This implies that the variability of costs must be less than $16.5 \%$. Neither number seems unreasonable, although further research on this issue is necessary (Armstrong et al. [24] discuss price caps under search and find that price caps might hurt the consumer because they soften competition).

(2) Many Marginal Costs. In this case, it is easy to see that our Lemma 1 can be generalized as follows. "The regulator should never set a price different from a possible value of the marginal cost." The proof is identical; for any price between two values of the marginal cost, apply the proof in the main text. The formulae showing welfare losses are less transparent, but the general principle applies: an expected social-welfare-maximizing regulator only considers either the marginal costs or the free market price to be an optimal choice. This may be called the generalized "price equals marginal costs" principle. See more on this in Point 3 below.

(3) Nonconstant Average Costs. Some of the markets in which price regulation has been used are characterized by large fixed costs (electricity, hospitals, etc.). Our paper can be interpreted as a model where fixed costs are known to the regulator and they are financed by nondistortionary taxation. The consideration of the Ramsey pricing (i.e., price equals average costs) would require different calculations, even though the principles established in this paper might be helpful. Next, let us consider increasing the marginal costs. Let $\rho=b=1, n=1$, and costs $c x^{2} / 2$ with $c=c_{H}$, $c_{L}$. We will assume that, once the price has been set by the regulator, firms are free to supply whatever quantity they find most convenient (Under constant returns to scale, if price equals marginal cost, the firm is indifferent to any output as long as it is sold in the market. Therefore, the implicit assumption in our previous analysis is that the regulator can choose any output from among those to which the firm is indifferent. When marginal costs are increasing, we have to be specific about the behaviour of firms). This implies that the monopolist supplies at a price that equals marginal costs unless this exceeds the regulated price of the product in the 
market. Consider that the regulator sets a price that equals, say, $c_{H}$. If the regulator decreases the price slightly, we have two effects. On the one hand, if the state is $c_{H}$, the output and social welfare decrease (following the "price equals marginal cost" rule). On the other hand, if the state is $c_{L}$, output and social welfare increase following the expansion of demand. By differentiating expected welfare and setting this to 0 , we find that the optimal price is

$$
p=\frac{A c_{H}\left[(1-\pi) c_{H} c_{L}+\pi\right]}{c_{H}^{2}\left(1+c_{L}\right)+\pi\left(1+c_{H}-c_{H}^{2}-c_{H}^{2} c_{L}\right)} .
$$

Note that, when $\pi=0$ (resp., 1), the regulated price is $A c_{L} /\left(1+c_{L}\right)$ (resp., $\left.A c_{H} /\left(1+c_{H}\right)\right)$, which results from equalizing the price to the marginal cost under state $c_{L}$ (resp., $c_{H}$ ). The general principle is that, in addition to the price equals marginal costs, there are other prices that might be optimal for the regulator because they are a good compromise between different states of the world.

(4) Other Forms of Demand. There are cases in which the percentage of welfare losses under free market depends on the state of the world. In this case, the results will not be as clean as those in this paper. Assume that the utility function of the representative consumer is cubic, written as $A x-b x^{2}-$ $d x^{3}-p x$. Thus, $p=A-2 b x-3 d x^{2}$ (see [25]). As in the main text, set $a \equiv A-c$. The optimal and the free market output are, respectively,

$$
\begin{gathered}
x^{O}=\frac{-b+\sqrt{b^{2}+3 a d}}{3 d}, \\
x^{C}=\frac{-b(1+1 / n)+\sqrt{b^{2}(1+1 / n)^{2}+3 a d+6(a d / n)}}{3 d+(6 d / n)} .
\end{gathered}
$$

Setting $b=d=n=1$, for simplicity, we see that, if $a$ tends to $0, \mathrm{PWL}^{C}$ tends to 0.25 , and, if $a$ tends to infinity, $\mathrm{PWL}^{C}$ tends to 0.23 . Thus, in this case, $\mathrm{PWL}^{C}$ depends on $a$.

\section{Appendix}

Proof of Lemma 3. We first show that $\operatorname{PWL}^{H}(\bullet, \rho, q)$ is increasing in $\beta$ if $\rho>0$ and decreasing in $\beta$ if $-1<\rho<0$.

$$
\begin{aligned}
& \frac{\partial \operatorname{PWL}^{H}(\beta, \rho, q)}{\partial \beta} \\
& =\frac{q \rho\left[(1+\rho) \beta^{1 / \rho}-\rho-1\right]+\beta^{(1+\rho) / \rho}(1+\rho)-\beta^{1 / \rho}(1+\rho)}{\rho^{2}\left(q+\beta^{(1+\rho) / \rho}\right)^{2}} \\
& =\frac{(1+\rho)\left[q \rho\left(\beta^{1 / \rho}-1\right)+\beta^{1 / \rho}(\beta-1)\right]}{\rho^{2}\left(q+\beta^{(1+\rho) / \rho}\right)^{2}} \mid \begin{array}{ll}
>0 & \text { if } \rho>0 \\
<0 & \text { if } \rho<0
\end{array}
\end{aligned}
$$

Note that, if $\rho>0, \beta>1$, if $\rho<0,0<\beta<1$, and $\beta^{1 / \rho}>1$ always.
We show that $\operatorname{PWL}^{Y}(\beta, \bullet, q)$ is decreasing in $\rho$ if $\rho>0$ and increasing if $-1<\rho<0$.

$$
\begin{aligned}
\frac{\partial \mathrm{PWL}^{H}(\beta, \rho, q)}{\partial \rho} & \beta^{(1+\rho) / \rho}\left(\frac{(\rho-1-\rho)}{\rho^{2}}\right) L N \beta\left(q+\beta^{(1+\rho) / \rho}-\beta^{(1+\rho) / \rho}\right) \\
& \times\left(q+\beta^{(1+\rho) / \rho}\right)^{-2} \\
& +\frac{\beta \beta^{(1+\rho) / \rho}\left((\rho-1-\rho) / \rho^{2}\right) L N \beta}{\left(q+\beta^{(1+\rho) / \rho}\right)^{2}} \\
& +(\beta-1)\left(q+\beta^{(1+\rho) / \rho}+\rho \beta^{(1+\rho) / \rho}\left((\rho-1-\rho) / \rho^{2}\right) L N \beta\right) \\
& \times\left(\rho^{2}\left(q+\beta^{(1+\rho) / \rho}\right)^{2}\right)^{-1} \\
= & -q \beta^{(1+\rho) / \rho} L N \beta-\beta \beta^{(1+\rho) / \rho} L N \beta+(\beta-1) \\
& \times\left(q+\beta^{(1+\rho) / \rho}(1-L N \beta / \rho)\right)\left(\rho^{2}\left(q+\beta^{(1+\rho) / \rho}\right)^{2}\right)^{-1} \\
& q\left(\beta^{(1+\rho) / \rho} L N \beta+1-\beta\right)+\beta^{(1+\rho) / \rho} \\
= & \frac{\times(\beta L N \beta-\beta+1+(\beta-1) L N \beta / \rho)}{\rho^{2}\left(q+\beta^{(1+\rho) / \rho}\right)^{2}} \mid \begin{array}{ll}
<0 & \forall \rho>0 \\
& \forall \rho \in(-1,0)
\end{array}
\end{aligned}
$$

If $\rho>0$ (resp., $<0$ ), this expression is negative: both parentheses in the numerator are positive (resp. negative) because they are increasing in $\beta$ and tend to 0 if $\beta \rightarrow 1$ :

$$
\begin{gathered}
\frac{\partial}{\partial \beta}\left(\beta^{(1+\rho) / \rho} L N \beta+1-\beta\right)=\frac{1+\rho}{\rho} \beta^{1 / \rho} L N \beta+\beta^{1 / \rho}-1>0, \\
\lim _{\beta \rightarrow 1}\left(\beta^{(1+\rho) / \rho} L N \beta+1-\beta\right)=0, \\
\frac{\partial}{\partial \beta}\left(\beta L N \beta-\beta+1+\frac{(\beta-1) L N \beta}{\rho}\right) \\
=L N \beta+\frac{L N \beta+(\beta-1) / \beta}{\rho}>0, \\
\lim _{\beta \rightarrow 1}\left(\beta L N \beta-\beta+1+\frac{(\beta-1) L N \beta}{\rho}\right)=0 .
\end{gathered}
$$

Finally, it is obvious that $\mathrm{PWL}^{H}$ decreases with $q$.

\section{Acknowledgment}

The authors thank Mark Armstrong, Carmen Beviá, Ángeles de Frutos, Natalia Fabra, Javier López-Cuñat, Lourdes Moreno, Roland Strausz, Ingo Vogelsang, and the participants in seminars at EARIE 2010, Istanbul, and Jornadas de Economía Industrial 2010, Madrid, for very useful comments and to María Hernández de Benito for support. The usual 
caveat applies. The first author acknowledges financial support from SEJ2005-06167/ECON.

\section{References}

[1] M. Armstrong and D. Sappington, "Recent developments in the theory of regulation," in Handbook of Industrial Organization, M. Armstrong and R. Porter, Eds., vol. 3, Elsevier, San Diego, Calif, USA, 2005.

[2] S. Shaffer, "A first-best regulatory tax for oligopoly," Journal of Regulatory Economics, vol. 1, no. 4, pp. 373-389, 1989.

[3] J. C. Kim and K. B. Chang, "An optimal tax/subsidy for output and pollution control under asymmetric information in oligopoly markets," Journal of Regulatory Economics, vol. 5, no. 2, pp. 183-197, 1993.

[4] M. Loeb and W. Magat, "A decentralized method for utility regulation," Journal of Law and Economics, vol. 22, no. 2, pp. 399-404, 1979.

[5] S. Schwermer, "Regulating oligopolistic industries: a generalized incentive scheme," Journal of Regulatory Economics, vol. 6, no. 1, pp. 97-108, 1994.

[6] S. H. O. Lee, "A note on regulating oligopolistic industries: a hierarchical model," Journal of Regulatory Economics, vol. 12, no. 1, pp. 91-97, 1997.

[7] D. Sappington and D. Sibley, "Regulating without cost information: the Incremental surplus subsidy scheme," International Economic Review, vol. 29, no. 2, pp. 297-306, 1988.

[8] J. López-Cuñat, "Regulating oligopolies," Revista Española de Economía, pp. 61-91, 1995.

[9] D. Baron and R. Myerson, "Regulating a monopolist with unknown costs,” Econometrica, vol. 50, no. 4, pp. 911-930, 1982.

[10] D. Kreps and J. Scheinkman, "Quantity pre-commitment and Bertrand competition yield Cournot outcomes," Bell Journal of Economics, vol. 14, no. 2, pp. 326-337, 1983.

[11] S. Littlechild, "Economic regulation of privatized water authorities," Report for the Department of Environment, HMSO, London, UK, 1986.

[12] S. P. Andersen and R. Renault, "Efficiency and surplus bounds in Cournot competition," Journal of Economic Theory, vol. 113, no. 2, pp. 253-264, 2003.

[13] J. Crémer and R. McLean, "Optimal selling strategies under uncertainty for a discriminating monopolist when demands are interdependent," Econometrica, vol. 53, no. 2, pp. 345-361, 1985.

[14] J. Crémer and R. McLean, "Full extraction of the surplus in Bayesian and dominant strategy auctions," Econometrica, vol. 56, no. 6, pp. 1247-1257, 1988.

[15] E. Maskin and J. G. Riley, "Auction design with correlated reservation values," Mimeo, 1980.

[16] R. B. Myerson, "Optimal auction design," Mathematics of Operations Research, vol. 6, no. 1, pp. 58-73, 1981.

[17] M. Armstrong, S. Cowan, and J. Vickers, Regulatory Reform: Economic Analysis and British Experience, MIT Press, Cambridge, Mass, USA, 1994.

[18] J. J. Laffont and J. Tirole, "Using cost observation to regulate firms," Journal of Political Economy, vol. 94, no. 3, pp. 614-641, 1986.

[19] I. Vogelsang, "Incentive regulation, investments and technological change," CESifo Working Paper no. 2964, 2010.

[20] J. J. Laffont and D. Martimort, The theory of Incentives: The Principal-Agent Model, Princeton University Press, Princeton, NJ, USA, 2001.
[21] R. Earle, K. Schmedders, and T. Tatur, "On price caps under uncertainty," Review of Economic Studies, vol. 74, no. 1, pp. 93$111,2007$.

[22] N. Fabra, N. H. von der Fehr, and M. A. de Frutos, "Market design and investment incentives," Working Paper, Carlos III University, 2009.

[23] S. Buehler, A. Burger, and R. Ferstl, "The investment effects of price caps under imperfect competition: a note," Economics Letters, vol. 106, no. 2, pp. 92-94, 2010.

[24] M. Armstrong, J. Vickers, and J. Zhou, "Consumer protection and the incentive to become informed," Journal of the European Economic Association, vol. 7, no. 2-3, pp. 399-410, 2009.

[25] J. P. McHardy, "Miscalculations of monopoly and oligopoly welfare losses with linear demand," Hull Economic Research Papers, 2000. 


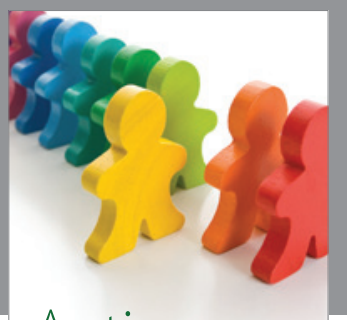

Autism

Research and Treatment
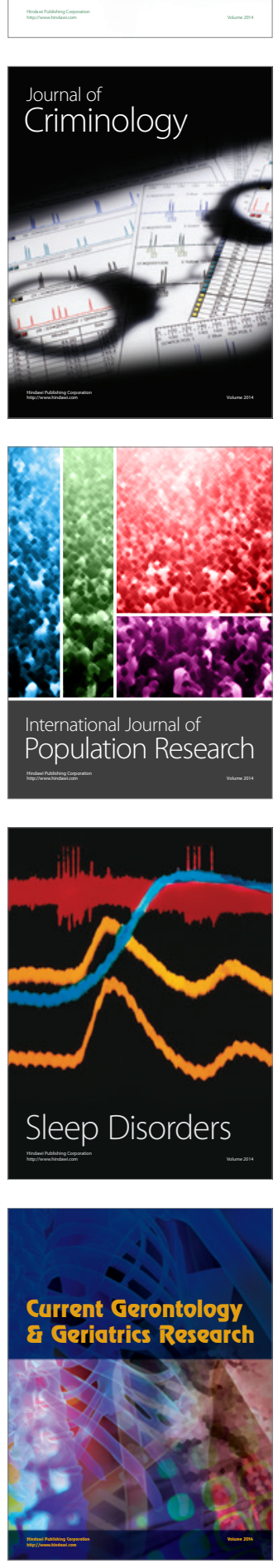
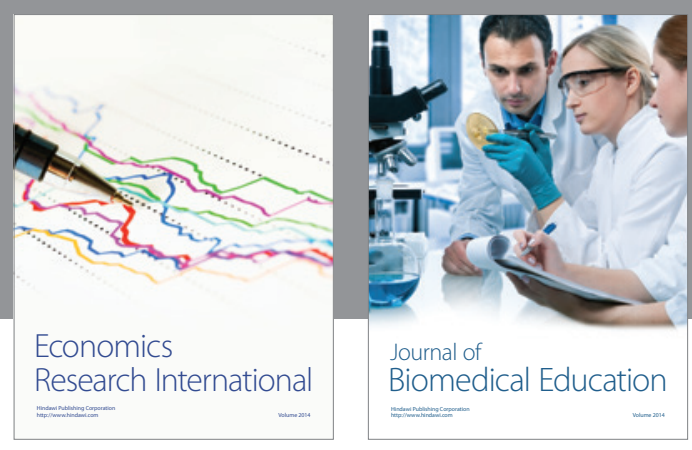

Journal of

Biomedical Education

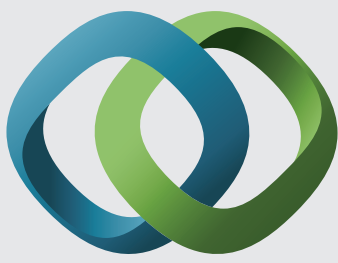

\section{Hindawi}

Submit your manuscripts at

http://www.hindawi.com
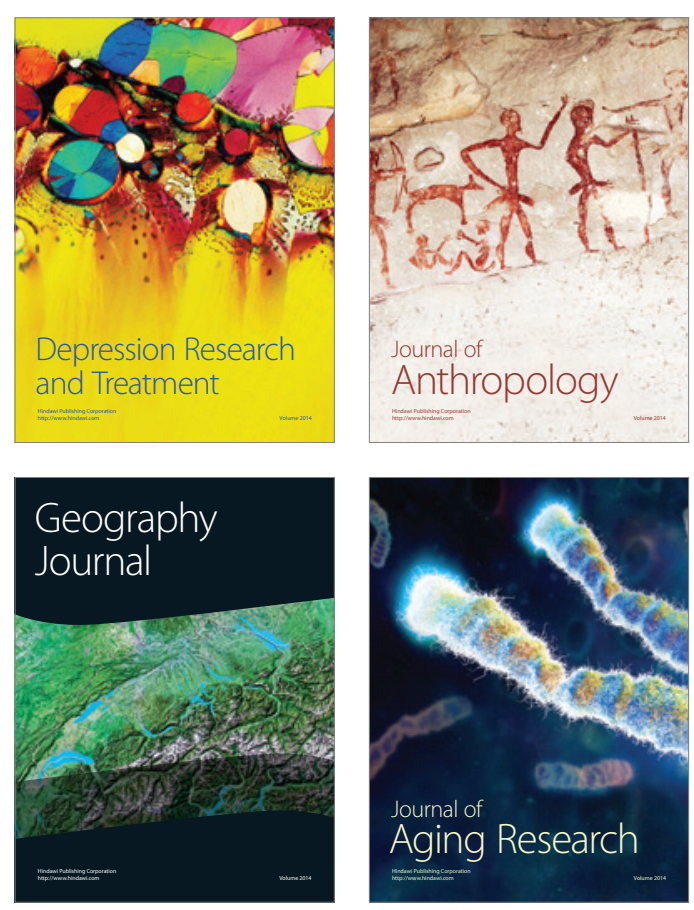

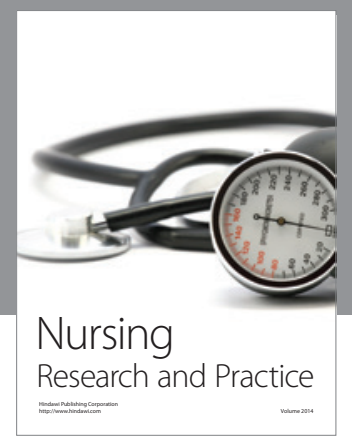

Nursing

Research and Practice

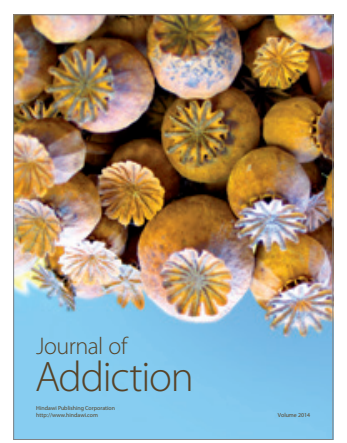

Child Development

Research

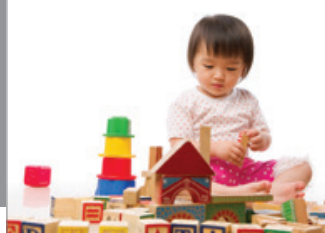

迥
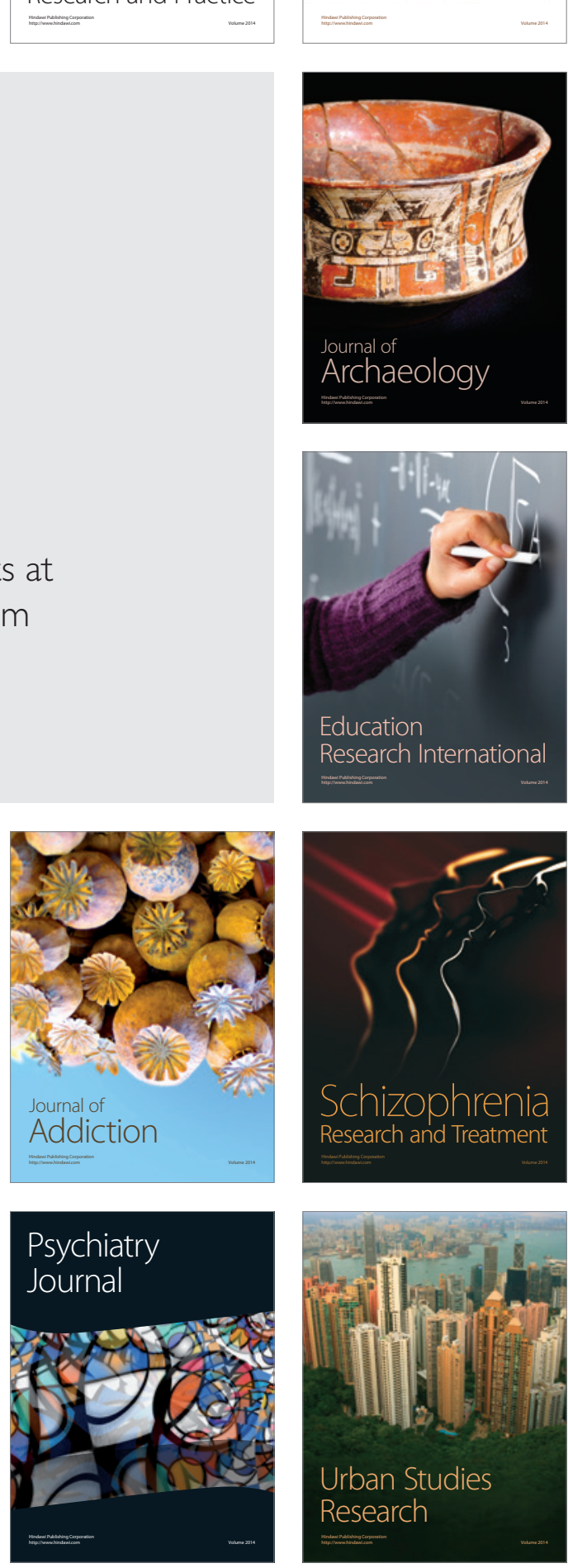